\title{
Integrating basic laboratory skills and clinical practice
}

Magdalena Coetzee, Mona-Liza Lottering, Somarie Grey \& Margaretha Viljoen

Editor - Over the years there have been many discussions on the inclusion of various types of extra skills teaching into medical curricula, on the integration of basic science and clinical aspects, and on the benefits or not of the intercalated BSc. Many thought-provoking ideas have been published in, amongst others, the Letters to the Editor and Really Good Stuff sections of Medical Education. ${ }^{1-3}$ Despite additional teaching of extra skills, there has been a severe reduction in the number of physiological and biochemical practicals included in medical curricula. This has not only led to the disappearance of analytical laboratory skills, but, and perhaps more significantly, to a decline in students' understanding of the need for proper collection and handling of biological specimens in laboratory evidence-based medicine. It has also led to a decline in training in how to carry out basic but valuable examinations of urine and blood specimens, which is particularly important for those who will eventually practise medicine in remote rural areas where no such facilities are available.

For several years now we have run an adaptation of the conventional case-based study that incorporates relatively simple laboratory skills into solving a problem. The exercise includes a written case history, laboratory analysis of blood and urine and, if needed to confirm the preliminary diagnosis, motivation for additional tests such as lung or liver functions, etc. The examination of the blood specimen includes establishing total white and red blood cell counts, haematocrit, haemoglobin levels, mean corpuscular volume, mean corpuscular haemoglobin, mean corpuscular haemoglobin concentration, microscopic red blood cell profile, and differential white blood cell counts. Urine examination includes the dipstick test and a microscopic evaluation of the centrifuged sediment for cells, casts, crystals, micro-organisms and any other abnormal constituents detectable by light microscopy. Manual techniques are used to show students that examinations can, if necessary, be performed without access to sophisticated laboratories. A final diagnosis

is made and a report written, which is expected to demonstrate that the student understands the relevant normal physiology and pathophysiology. Students are guided throughout the problem-solving experience and grades are allocated for the different activities. The outcome is presented in a mini-symposium where students are evaluated on their presentation skills and the way they handle questions.

Although we offer this course to BSc and BMedSci students - of whom some apply to enter medicine and some do not - we are convinced that a similar course would be of benefit to all medical students and might contribute to their understanding of evidence-based medicine.

\section{References}

1. HudsonJN. A further example of paired-teacher lecturing to link theory to practice. Med Educ

2005;39:1254

2 AghaR, SinghG. Studying for an intercalated BSc. Med Educ 2003;37:839.

3 BrownellA, ed. Really Good Stuff: new ideas in medical education. Med Educ 2003;37:473-89. 